\title{
Elusive structure of helium trimers
}

\author{
Petar Stipanović ${ }^{1}$, Leandra Vranješ Markić ${ }^{1}$ and Jordi Boronat ${ }^{2}$ \\ ${ }^{1}$ Faculty of Science, University of Split, Rudera Boškovića 33, HR-21000 Split, \\ Croatia \\ ${ }^{2}$ Departament de Física, Campus Nord B4-B5, Universitat Politècnica de Catalunya, \\ E-08034 Barcelona, Spain \\ E-mail: pero@pmfst.hr
}

April 2016

\begin{abstract}
Over the years many He-He interaction potentials have been developed, some very sophisticated, including various corrections beyond Born-Oppenheimer approximation. Most of them were used to predict properties of helium dimers and trimers, examples of exotic quantum states, whose experimental study proved to be very challenging. Recently, detailed structural properties of helium trimers were measured for the first time, allowing a comparison with theoretical predictions and possibly enabling the evaluation of different interaction potentials. The comparisons already made included adjusting the maxima of both theoretical and experimental correlation functions to one, so the overall agreement between theory and experiment appeared satisfactory. However, no attempt was made to evaluate the quality of the interaction potentials used in the calculations. In this work, we calculate the experimentally measured correlation functions using both new and old potentials, compare them with experimental data and rank the potentials. We use diffusion Monte Carlo simulations at $T=0$, which give within statistical noise exact results of the ground state properties. All models predict both trimers ${ }^{4} \mathrm{He}_{3}$ and ${ }^{4} \mathrm{He}_{2}{ }^{3} \mathrm{He}$ to be in a quantum halo state.

PACS numbers: 02.70.Ss, 36.40.-c, 67.90.+z
\end{abstract}

Keywords: helium trimers, structural properties, quantum halo states, interaction potential, ground-state, quantum Monte Carlo 


\section{Introduction}

Helium is the second lightest and the second most abundant element in the observable universe. Only two electrons in a closed 1s shell make helium the most unpolarizable element. Hence, two He atoms experience extremely weak van der Waals interaction. As a consequence of that and a small atomic mass, He remains liquid under normal pressure, even when the absolute temperature goes to zero, and becomes superfluid at low temperatures. Microscopic superfluidity was noticed [1] and recently reviewed, together with other quantum effects of He clusters and droplets, by Toennies [2].

Several theoretical calculations predicted the existence of the smallest stable helium clusters. Different mass and spin nature of helium isotopes, ${ }^{4} \mathrm{He}$ and ${ }^{3} \mathrm{He}$, affect the stability of helium clusters. Bound ground state of ${ }^{4} \mathrm{He}_{N}$ clusters was predicted [3] for any $N>1$, while $M \geq 30$ atoms are needed [4] to form a stable cluster ${ }^{3} \mathrm{He}_{M}$. If one ${ }^{4} \mathrm{He}$ is added to ${ }^{3} \mathrm{He}_{M}$, a mixed cluster becomes stable [5, 6] for $M \geq 20$, while addition of two ${ }^{4} \mathrm{He}$ atoms reveals magic numbers, $M=1$ being the smallest one.

Experimental confirmations of the smallest helium cluster, about twenty years ago, stimulated their theoretical analysis. Moreover, the first experimental evidences of the fragile dimer ${ }^{4} \mathrm{He}_{2}$ seemed to be elusive. Its existence was predicted with a binding energy of $\sim 10^{-7} \mathrm{eV}$, which is negligible when compared to the binding of the dimer $\mathrm{H}_{2}(\sim 5 \mathrm{eV})$, which is only one step away in the periodic table. Due to its weak bond, traditional particle probes of atomic structure, i.e., microwave, infrared, and visible light spectroscopy, x-ray diffraction, and electron scattering, were doomed to fail. Finally, the stability of the dimer ${ }^{4} \mathrm{He}_{2}$ was confirmed by mass spectroscopy [7, 8], while dimer and trimer of ${ }^{4} \mathrm{He}$ were detected using diffraction from the nanoscale grating [9, 10]. More precise measurements were realized half a decade later by means of diffraction of helium clusters from a $100 \mathrm{~nm}$ period transmission grating. Analyzing these measurements the mean interparticle distance $\langle r\rangle$ and binding energy $E_{2}$ of ${ }^{4} \mathrm{He}_{2}$ were found [1] to be:

$$
\begin{aligned}
& E_{2}^{\prime}=-1.1_{-0.3}^{+0.2} \mathrm{mK}, \\
& \left\langle r^{\exp }\right\rangle=52(4) \AA .
\end{aligned}
$$

In 2005, mixed helium clusters ${ }^{4} \mathrm{He}_{N}{ }^{3} \mathrm{He}_{M}$ with up to 8 atoms, including ${ }^{4} \mathrm{He}_{2}{ }^{3} \mathrm{He}$, were identified using nondestructive transmission grating diffraction [12]. Recently, Coulomb explosion imaging of diffracted clusters ${ }^{4} \mathrm{He}_{3}$ and ${ }^{4} \mathrm{He}_{2}{ }^{3} \mathrm{He}$ was reported [13]. From these experimental data, distributions of interparticle separations and distributions of angles in triangles formed by ${ }^{4} \mathrm{He}_{3}$ and ${ }^{4} \mathrm{He}_{2}{ }^{3} \mathrm{He}$ clusters were extracted. Furthermore, it was confirmed that the ground state of ${ }^{4} \mathrm{He}_{2}{ }^{3} \mathrm{He}$ is a quantum halo state, which is usually defined as a weakly bound state whose size extends far into the classically forbidden regions [14, 15].

Universal scaling of energy and size of exotic dimers and trimers in quantum halo states was recently studied [16]. It was predicted for a particular realistic interaction potential that both ${ }^{4} \mathrm{He}_{3}$ and ${ }^{4} \mathrm{He}_{2}{ }^{3} \mathrm{He}$ can be classified as quantum halo states, although ${ }^{4} \mathrm{He}_{3}$ was very close to the usually defined limit for these states. 
The trimer ${ }^{4} \mathrm{He}_{3}$, which is weakly bound under natural conditions, has been longly considered an ideal candidate for observing Efimov states [17]. In an Efimov state, an infinite series of stable three-body states, with geometrically spaced binding energies, occurs when a third particle is added to a pair of bosons that are on the edge of binding. After a long and continued research, finally, few months ago, the Efimov state was detected [18, 19] in the only excited state of ${ }^{4} \mathrm{He}_{3}$ by means of Coulomb explosion imaging of masses selected by transmission grating diffraction.

If the interaction potential between He atoms is accurately known, then all the ground state properties of He clusters can be precisely predicted using quantum simulation methods. Therefore, the accuracy of theoretical predictions depends on the interaction potential model, which can be evaluated only by the comparison with experimental observations. Over the years, with emergence of more precise measurements, it becomes important to know this interatomic potential with increasing accuracy. In fact, the determination of interaction potential models has been the subject of extensive activity; some of them are given in $[20,21,22,23,24,25,26,27,28$.

Despite of continued efforts, the exact value of the binding energy $E_{2}$ is still being disputed due to discrepancies between the theoretical predictions and the experimental measurements. From the theoretical side, several sophisticated nonrelativistic BornOppenheimer $\left(V_{\mathrm{BO}}\right)$ helium dimer pair potentials were developed, such as those given in [23, 24, 26]. Recently, Przybytek et al calculated [27] and Cencek et al analyzed [28] the main post- $V_{\mathrm{BO}}$ physical effects, i.e., the adiabatic, relativistic, quantum electrodynamics, and retardation contributions. But none of these post- $V_{\mathrm{BO}}$ corrections predicted, within error bars, the experimental result (1) for the ${ }^{4} \mathrm{He}$ dimer energy. Threebody effects were theoretically discussed [20, 22, 25] as well, but experimental verification of their relevance is also missing. Recently, newly applied Coulomb explosion imaging by Voigtsberger et al [13] and Kunitski et al [18] provided direct information on the structure of helium trimers. This opened up the question on how predictions obtained with different potentials fit in those recently published distributions [13, 18].

Theoretical predictions of energy and structural properties of course depend on the model of interaction potential. Differences are especially noticeable in small mass clusters due to significant cancellation between kinetic and potential energies. Stipanović et al [29] evaluated binding energies and structural properties of mixed clusters of ${ }^{4} \mathrm{He}$ and spin-polarized tritium $(\mathrm{T} \downarrow)$ using different potential models and concluded that differences are lowered with the increase of the cluster size (number of atoms). Significant differences were noticed in case of the trimer ${ }^{4} \mathrm{He}_{2} \mathrm{~T} \downarrow$, e.g., when the most accurate ${ }^{4} \mathrm{He}-\mathrm{T} \downarrow$ model is replaced by the frequently used model, the binding energy is reduced by almost $80 \%$, which is also reflected in different distribution functions. Therefore, helium trimers, with similarly weak binding, seem to be an ideal system to test different corrections of helium potential models.

In this work, we report how different $V_{\mathrm{BO}}$ potentials and their corrections affect the ground-state energy and structural properties of the helium dimer ${ }^{4} \mathrm{He}_{2}$ and trimers ${ }^{4} \mathrm{He}_{3}$ and ${ }^{4} \mathrm{He}_{2}{ }^{3} \mathrm{He}$. In section 2, we report the selected potential models and corrections. We 
also introduce the diffusion Monte Carlo method (DMC) [30] and discuss the trial wave functions used for importance sampling. Section 3 reports the results obtained by the DMC simulations. We compare our results with other theoretical work and particularly with experimentally determined distribution functions. In addition, we report a ranking of interaction potentials according to the agreement with experiments. Finally, section 4 comprises a summary of the work and an account of the main conclusions.

\section{Method}

To evaluate the effect of potential models on the binding energy and size of these weakly bound and extended clusters a very accurate calculation needs to be done. This goal can be achieved using the DMC method with pure estimators [31]. First, we compare different potentials and select model types and corrections that are expected to produce significant effects on the physical properties of the studied He clusters.

\subsection{Interaction potential models}

We modeled the interaction of $\mathrm{He}$ atoms in clusters ${ }^{4} \mathrm{He}_{3}$ and ${ }^{4} \mathrm{He}_{2}{ }^{3} \mathrm{He}$ by potentials that were obtained using different methods and levels of approximations.

Among frequently used $V_{\mathrm{BO}}$ potentials, we selected three forms for the entire van der Waals He-He pair potential curve. The first is the semi-empirical HFDB form given by Aziz et al [23] who adapted the B-type of Hartree-Fock model with damped dispersion (HFD) to experimental and theoretical results. The second is derived from perturbation theory by Tang, Toennies and Yiu (TTY) 24] who gave a relatively simple analytical expression. The most sophisticated theoretical $V_{\mathrm{BO}}$-model was published few years ago by Jeziorska et al [26]. They combined supermolecular (SM) data and the symmetryadapted perturbation theory (SAPT) in order to obtain a fitted analytic function for the He-He potential (SAPTSM) and for its error bars $(\varsigma)$. Using these fits, they obtained a well depth of $V_{\mathrm{m}}=-11.006(4) \mathrm{K}$ at the equilibrium distance $r_{\mathrm{m}}=5.608(12) a_{0}$, which is shown by the second vertical line in figure 1, while the first (on the left) vertical line separates the attractive and repulsive parts of the SAPTSM potential.

Three models of three-body interactions were tested: the Axilrod-Teller [20] (V3AT) and Brunch-McGee [22] (V3BM) potentials, analyzed in [30], as well as the more recent fit [25] of a triple dipole damping function and the three-body exchange interaction intensity (V3DDDJ).

We also studied different corrections $\Delta V$ to the $V_{\mathrm{BO}}$-models. Recently, corrections

of the $V_{\mathrm{BO}}$-model SAPTSM were calculated by Przybytek et al [27] who included leading order coupling of the electronic and nuclear motion, i.e., adiabatic corrections, relativistic and quantum electrodynamics contributions (ARQ). Additionaly, they calculated Casimir-Polder [21] retardation effects appropriate for each level of correction (adiabatic, adiabatic+relativistic, and so on). They also computed dissociation energies for ${ }^{4} \mathrm{He}-{ }^{4} \mathrm{He}$ using those types of corrections, and showed that some of them cancel each 


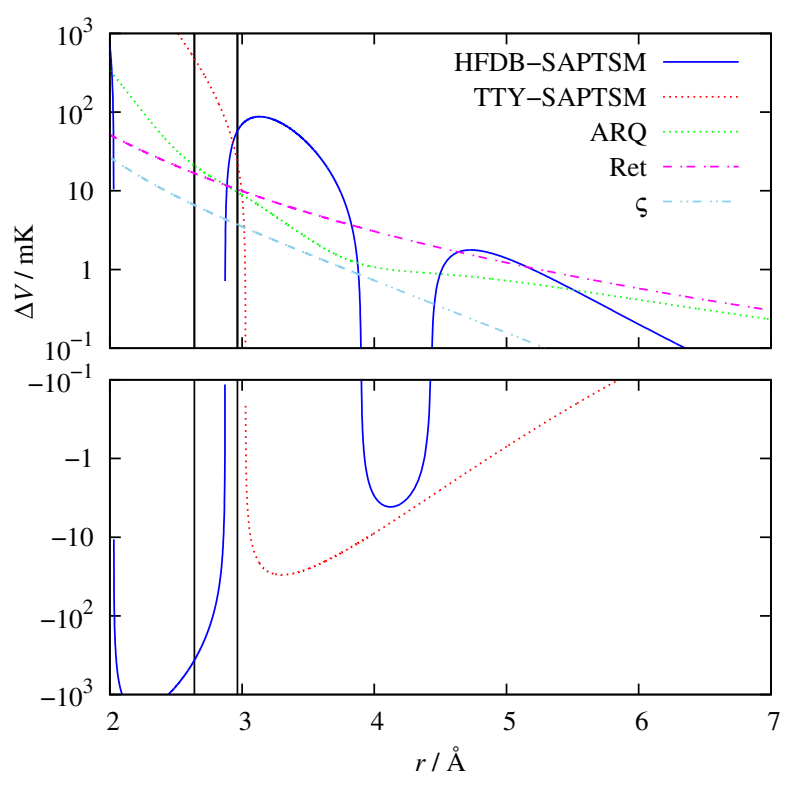

Figure 1. Difference of He-He potentials HFDB [23] and SAPTSM [26]; TTY 24 and SAPTSM; SAPTSM-adiabatic correction and relativistic and quantum electrodynamics contributions (ARQ), SAPTSM-error $\varsigma$ and SAPTSM-retardation correction from 27] are compard on logarithmic scale as a function of separation $r$ between helium atoms. Vertical lines separate the attractive and repulsive parts of SAPTSM (left) and designate the equilibrium distance (right).

other. The $V_{\mathrm{BO}}$-dissociation energy $E=1.718 \mathrm{mK}$ is changed the most by including among all $V_{\mathrm{BO}}$-corrections only the retardation term appropriate for the $V_{\mathrm{BO}}$ interaction potential (Ret); in that case it is lowered approximately by $10 \%$. On the one hand, by including all corrections, i.e. when using the complete SAPTSM+ARQ retarded potential (PCKLJS - the authors [27] acronym), the dissociation energy is 1.62(3) $\mathrm{mK}[28]$.

In order to compare the selected $V_{\mathrm{BO}}$ potentials and their corrections, in figure 1 we plot the differences HFDB-SAPTSM, TTY-SAPTSM, together with ARQ and Ret correction, and the error of the SAPTSM potential $\varsigma$. As the separation of He atoms $r$ increases, both corrections (ARQ, Ret, $\varsigma$ ) and oscillating differences (HFDB-SAPTSM, TTY-SAPTSM) decay very fast (notice the logarithmic scale). All plotted differences are by absolute value higher than $\varsigma$, so it is interesting to investigate whether their impact on the cluster properties could be discerned in experiments.

As discussed in section 3, experimentally measured trimer properties suggest even weaker potentials than the previously mentioned models. This prompted us to test if small changes in the interaction potential model could account for the experimental findings. During the potential model construction, dispersion coefficients $C_{i}$ are usually attenuated by fitting damping functions to experimental or theoretical data. Simplifying this procedure, lowering only the coefficient $C_{6}$ used in the HFDB model, we tried to obtain a new potential that could predict structural properties of He trimers more 


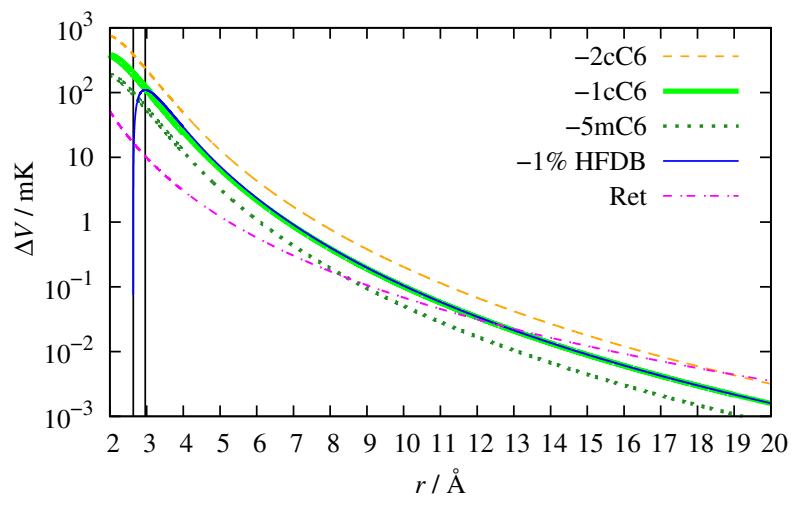

Figure 2. Modifications -rC6 of HFDB [23, where $\mathrm{r}$ denotes how much the parameter $C_{6}$ is reduced: $2 \mathrm{c}(0.02), 1 \mathrm{c}(0.01)$ and $5 \mathrm{~m}(0.005),-1 \%$ of HFDB and retardation correction Ret [27] are compared on logarithmic scale as a function of separation $r$ between helium atoms. Vertical lines separate the attractive and repulsive parts of HFDB (left) and show the equilibrium distance (right).

accurately than the previously mentioned corrections. To this end, we constructed three models HFDB-rC6 where ' $r$ ' denotes the factor by which the parameter $C_{6}$ is reduced: $0.02(2 \mathrm{c}), 0.01(1 \mathrm{c})$ and $0.005(5 \mathrm{~m})$, e.g., $-5 \mathrm{mC} 6$ means $C_{6} \rightarrow C_{6}-0.005 C_{6}$. Comparison of -rC6 corrections with Ret is given in figure 2. Vertical lines stand for the starting of attractive part (on the left) and position of HFDB minimum (on the right). At short distances $\Delta V$ from -rC6 models are more significant than Ret, while at long range Ret becomes dominant. On the same figure, we plot the reversed percentage of HFDB. It serves to show that the correction -1cC6 (wide solid line) corresponds to reducing the attractive part of HFDB by $1 \%$ (thin solid line).

\subsection{Diffusion Monte Carlo method}

For the study of clusters at zero temperature we use the DMC method. The DMC method solves stochastically the Schrödinger equation written in imaginary time $\tau=$ $i t / \hbar$,

$$
-\frac{\partial \Psi(\boldsymbol{R}, \tau)}{\partial \tau}=\left(H-E_{\mathrm{r}}\right) \Psi(\boldsymbol{R}, \tau)
$$

applying reasonable approximations for the Green's function when $\Delta \tau \rightarrow 0$. $E_{\mathrm{r}}$ is a constant acting as a reference energy and $\boldsymbol{R} \equiv\left(\boldsymbol{r}_{1}, \boldsymbol{r}_{2}, \boldsymbol{r}_{3}\right)$ collectively denotes the positions of the trimer constituents. The Hamiltonian $H$ for the helium trimer is

$$
H=-\sum_{i=1}^{3} \frac{\hbar^{2}}{2 m_{i}} \nabla_{i}^{2}+\sum_{\substack{i, j=1 \\ i<j}}^{3} V_{2}\left(r_{i j}\right)+\left\{V_{3}(\boldsymbol{R})\right\}
$$

where $V_{2}$ denotes the He-He pair potential and $V_{3}$ the three-body interaction, if used. Roudnev and Cavagnero [32] stressed the sensitivity of benchmarked dimer and trimer properties to fundamental constants. Thus we used the best available data from the 
NIST database, with constant $\hbar^{2} m^{-1}$ for ${ }^{4} \mathrm{He}$ and ${ }^{3} \mathrm{He}$ equal to $12119.28157 \mathrm{mK} \AA^{2}$ and $16083.62212 \mathrm{mK} \AA^{2}$, respectively.

In order to reduce the variance of the calculation to a manageable level, a common practice is to use importance sampling by introducing a guiding wave function $\psi(\mathbf{R})$. Specifically, the Schrödinger equation (2) is rewritten for the mixed distribution $\Phi(\boldsymbol{R}, \tau)=\Psi(\boldsymbol{R}, \tau) \psi(\boldsymbol{R})$. Within the Monte Carlo framework, $\Phi(\boldsymbol{R}, \tau)$ is represented by a set of walkers $\{\boldsymbol{R}\}$. In the limit $\tau \rightarrow \infty$, for long simulation times, providing that $\psi(\mathbf{R})$ is not orthogonal to the exact ground-state wave function $\psi_{0}(\boldsymbol{R})$, and has non-zero overlap with $\psi_{0}(\boldsymbol{R})$ in all regions where $\psi_{0}(\boldsymbol{R}) \neq 0$, only the lowest energy eigenfunction survives, $\Psi(\boldsymbol{R}, \tau) \rightarrow \psi_{0}(\boldsymbol{R})$. This allows the calculation of the ground state expectation values by stochastic sampling. Apart from statistical uncertainties, the ground-state energy $E$ of an $N$-body bosonic system is exactly calculated, which applies also to the calculations in the present work because they involve no more than one fermion.

To guide the diffusion process, we used trial wave function optimized using the variational Monte Carlo (VMC) method, minimizing the energy and its variance. The trial wave function is of Jastrow type, $\psi\left({ }^{4} \mathrm{He}_{3}\right)=F_{4}\left(r_{12}\right) F_{4}\left(r_{13}\right) F_{4}\left(r_{23}\right)$ and $\psi\left({ }^{4} \mathrm{He}_{2}{ }^{3} \mathrm{He}\right)=F_{44}\left(r_{12}\right) F_{43}\left(r_{13}\right) F_{43}\left(r_{23}\right)$, i.e. a product of two-body correlation functions

$$
F_{i}(r)=\frac{1}{r} \exp \left[-\left(\frac{\alpha_{i}}{r}\right)^{\gamma_{i}}-s_{i} r\right]
$$

where $r$ is the interparticle distance and $i=4,44,43$. Variational parameters $\alpha_{i}$ and $\gamma_{i}$ describe short-range correlations, while $s_{i}$ is used for the long-range ones. The optimization of the trial wave functions was done for all clusters and all models. Due to a small change in the VMC energy when model-optimal parameters were swapped, e.g., less than $1 \mathrm{mK}$ for bare $V_{\mathrm{BO}}$, the same parameters were used for a particular cluster in all further DMC simulations. Only in the case of -rC6 corrections, the parameters $s_{i}$ were slightly lowered. In the case of the ${ }^{4} \mathrm{He}_{3}$ cluster, parameters $\alpha_{4}=2.82 \AA, \gamma_{4}=4.14$ and $s_{4}=0.027 \AA^{-1}$ lowered the VMC energy to $86 \%-92 \%$ of the DMC energy. The difference between VMC and DMC results increased when one ${ }^{4} \mathrm{He}$ was swapped by ${ }^{3} \mathrm{He}$, e.g., HFDB-optimal parameters $\alpha_{44}=2.79 \AA, \gamma_{44}=4.21, s_{44}=0.017 \AA^{-1}, \alpha_{43}=2.87 \AA$, $\gamma_{43}=3.74$ and $s_{43}=0.0006 \AA^{-1}$ in VMC returned $40 \%$ of the DMC energy. Additional tests were performed with significant changes in parameters to ensure that the guiding wave function did not introduce any energy bias.

We used a DMC method [30] which is accurate to second order in the time step $\Delta \tau, E_{\mathrm{DMC}}(\Delta \tau)=E+k_{E}(\Delta \tau)^{2}$. Both the time step dependence and the mean walker population were studied carefully in order to eliminate any bias. For both trimers, ${ }^{4} \mathrm{He}_{3}$ and ${ }^{4} \mathrm{He}_{2}{ }^{3} \mathrm{He}, 5000$ walkers proved to be enough. The DMC energies $E_{\mathrm{DMC}}(\Delta \tau)$ were calculated for different time steps (from $4 \times 10^{-4} \mathrm{~K}^{-1}$ to $16 \times 10^{-4} \mathrm{~K}^{-1}$ ) and the final results were derived by extrapolation to zero time step.

The expectation value of an operator which does not commute with the Hamiltonian $H$ can be accurately calculated using pure estimators [31]. For the average potential energy $E_{\mathrm{p}}$, mean square root of pair distances $r_{i j}$, density profiles $\rho(r)$, the pair $P(r)$ and 
angular distribution $P(\vartheta)$ functions in the clusters we verified that the chosen block size is large enough to guarantee asymptotic offspring, i.e., to correct the bias coming from the choice of the trial wave function. All presented results were obtained using 90000 steps per block, although some properties converged even for 3 times smaller block sizes.

\section{Results}

\subsection{Binding energies}

In table 1, we present detailed calculations of the helium dimer energy and the scattering parameters obtained using selected potentials, in combination with different corrections. Potential models are presented in descending order, from the strongestbinding model SAPTSM to the weakest one HFDB-2cC6. We did not consider all possible combinations of corrections because of tiny differences between them. Namely [27, 28], adiabatic correction strengthens the binding, which is weakened after adding appropriate retardation correction, further weakened after including relativistic corrections and strengthened applying level appropriate retardation. Again, this is increased including quantum electrodynamics effects, but decreased applying level appropriate retardation. Therefore, just mentioned corrections would oscillate approximately between values obtained by SAPTSM and SAPTSM+Ret, with only the adiabatic correction being outside those limits, but very close to SAPTSM.

The ground-state binding energy and scattering length of ${ }^{4} \mathrm{He}_{2}$, obtained using the SAPTSM and SAPTSM+Ret potentials, are in agreement with values given in [26, 27, 28]. For the case of the TTY and HFDB potentials our results agree with those reported in [24, 23, 32. Using the PCKLJS potential, Cencek et al [28] obtained $E_{2}=-1.62(3) \mathrm{mK},\langle r\rangle=47.1(5) \AA$ and $a_{\mathrm{s}}=90.4(9) \AA$, values which are between the predictions of models HFDB and SAPTSM+Ret. In contrast to the PCKLJS result, TTY, TTY+Ret, and HFDB-5mC6 predict the dimer binding given in (1a) within error bars. Reduction of $C_{6}$ in HFDB model by $2 \%$ decreases too much the binding energy, almost to the threshold of the dimer binding.

Cencek et al [28] commented that the value (1a) cannot be considered reliable because it was estimated using the pretty rough approximation $E_{2}^{\exp }=-\hbar^{2} /\left(m a_{\mathrm{s}}^{2}\right)=$ $-\hbar^{2} /\left(4 m\langle r\rangle^{2}\right)$. Furthermore, they suggested that a better analysis could be made weakening the PCKLJS potential by adding 9.6ৎ to it. In this way, they could obtain $(1 \mathrm{~b})$. Using weakened potentials they estimated

$$
\begin{aligned}
& E_{2}^{\prime \prime}=-1.30_{-0.25}^{+0.19} \mathrm{mK}, \\
& a_{\mathrm{s}}^{\prime \prime}=100.2_{-7.9}^{+8.0} \AA .
\end{aligned}
$$

which are in agreement with the major part of the theoretical estimates given in table1: TTY, SAPTSM+Ret and additional models used only in this work HFDB+Ret, TTY+Ret and HFDB-5mC6.

All selected potential models predict different ${ }^{4} \mathrm{He}-{ }^{4} \mathrm{He}$ binding energies $E_{2}$ and scattering lengths $a_{\mathrm{s}}$. However, the best predictor cannot be selected due to the large 
Table 1. Scattering length $a_{\mathrm{s}}$, effective range $r_{\mathrm{e}}$ and binding energy $E_{2}$ for helium pairs ${ }^{4} \mathrm{He}-{ }^{4} \mathrm{He}$ and ${ }^{4} \mathrm{He}-{ }^{3} \mathrm{He}$ estimated using different potential models (for details see section 2.1).

\begin{tabular}{|c|c|c|c|c|}
\hline Pair & Potential model & $a_{\mathrm{s}} / \AA$ & $r_{\mathrm{e}} / \AA$ & $E_{2} / \mathrm{mK}$ \\
\hline \multirow{9}{*}{ 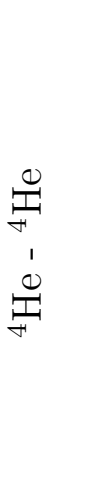 } & SAPTSM & 87.544 & 7.274 & -1.73 \\
\hline & HFDB & 88.430 & 7.276 & -1.69 \\
\hline & SAPTSM+Ret & 91.816 & 7.287 & -1.56 \\
\hline & HFDB+Ret & 92.803 & 7.290 & -1.53 \\
\hline & TTY & 99.588 & 7.328 & -1.32 \\
\hline & TTY+Ret & 105.204 & 7.342 & -1.18 \\
\hline & HFDB-5mC6 & 108.666 & 7.348 & -1.10 \\
\hline & HFDB-1cC6 & 141.592 & 7.422 & -0.64 \\
\hline & HFDB-2cC6 & 373.644 & 7.576 & -0.09 \\
\hline \multirow{9}{*}{ 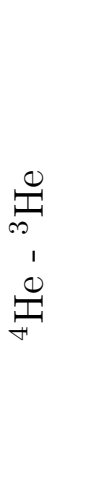 } & SAPTSM & -18.234 & 9.749 & - \\
\hline & HFDB & -18.204 & 9.751 & - \\
\hline & SAPTSM+Ret & -17.966 & 9.759 & - \\
\hline & HFDB+Ret & -17.936 & 9.761 & - \\
\hline & TTY & -17.593 & 9.847 & - \\
\hline & TTY+Ret & -17.339 & 9.857 & - \\
\hline & HFDB-5mC6 & -17.265 & 9.878 & - \\
\hline & HFDB-1cC6 & -16.403 & 10.009 & - \\
\hline & HFDB-2cC6 & -14.873 & 10.282 & - \\
\hline
\end{tabular}

uncertainties in energies $(1 a)$ and $(5 a)$.

As expected, due to smaller ${ }^{3} \mathrm{He}$ mass, none of the potentials predict binding of the mixed helium pair.

In agreement with the literature, only two isotopic combinations are predicted to form trimers: ${ }^{4} \mathrm{He}_{3}$ and ${ }^{4} \mathrm{He}_{2}{ }^{3} \mathrm{He}$. Trimers can be classified according to the number of bound two-body subsystems. Borromean [33], tango [34], samba [35] and all-bound type have zero, one, two and all three dimer subsystems bound, respectively. All potential models considered here classify trimers ${ }^{4} \mathrm{He}_{3}$ and ${ }^{4} \mathrm{He}_{2}{ }^{3} \mathrm{He}$ as all-bound and tango trimer type, respectively. Their ground-state energies predicted by different potential models are reported in table 2. DMC statistical errors $\sigma_{E}$ are given in brackets while \pm denote differences in binding when SAPTSM potential is increased or reduced by the error $\varsigma$. Thus, DMC statistical errors are few times smaller than errors which originate from SAPTSM uncertainty. Differences in predicted trimer energies between the weakest and the strongest studied $V_{\mathrm{BO}}$ model are of the order $6 \mathrm{mK}(3 \mathrm{mK})$ in the case of ${ }^{4} \mathrm{He}_{3}$ $\left({ }^{4} \mathrm{He}_{2}{ }^{3} \mathrm{He}\right)$, which is less than $5 \%$ of the whole binding energy of ${ }^{4} \mathrm{He}_{3}$, but amounts to approximately $20 \%$ of the binding energy of ${ }^{4} \mathrm{He}_{2}{ }^{3} \mathrm{He}$. Retardation correction lowers the binding energies of ${ }^{4} \mathrm{He}_{3}$ and ${ }^{4} \mathrm{He}_{2}{ }^{3} \mathrm{He}$ by about $2 \mathrm{mK}$ and $1 \mathrm{mK}$, respectively. All 
Elusive structure of helium trimers

Table 2. Average potential energy $E_{\mathrm{p}}$ and binding energy $E_{3}$ of helium trimers ${ }^{4} \mathrm{He}_{3}$ and ${ }^{4} \mathrm{He}_{2}{ }^{3} \mathrm{He}$ estimated using different potential models (for details see section 2.1).

\begin{tabular}{|c|c|c|c|}
\hline Trimer & Potential model & $E_{\mathrm{p}} / \mathrm{mK}$ & $E_{3} / \mathrm{mK}$ \\
\hline \multirow{12}{*}{$\underbrace{\infty}_{\forall}$} & TTY & $-1786(7)$ & $-126.36(39)$ \\
\hline & TTY+Ret & $-1774(6)$ & $-124.20(26)$ \\
\hline & HFDB & $-1835(4)$ & $-133.24(17)$ \\
\hline & $\mathrm{HFDB}+\mathrm{V} 3 \mathrm{AT}$ & $-1821(7)$ & $-132.46(30)$ \\
\hline & $\mathrm{HFDB}+\mathrm{V} 3 \mathrm{BM}$ & $-1834(6)$ & $-132.95(25)$ \\
\hline & HFDB+V3DDDJ & $-1829(4)$ & $-132.76(14)$ \\
\hline & HFDB+Ret & $-1814(5)$ & $-130.47(18)$ \\
\hline & $\operatorname{SAPTSM} \pm \varsigma$ & $-1832(8)$ & $-133.37(24) \pm_{0.7}^{0.4}$ \\
\hline & SAPTSM+Ret $\pm \varsigma$ & $-1823(6)$ & $-130.82(16) \pm_{0.8}^{0.5}$ \\
\hline & HFDB-2cC6 & $-1552(4)$ & $-94.07(28)$ \\
\hline & HFDB-1cC6 & $-1691(5)$ & $-112.85(12)$ \\
\hline & HFDB-5mC6 & $-1765(9)$ & $-122.82(13)$ \\
\hline \multirow{12}{*}{ 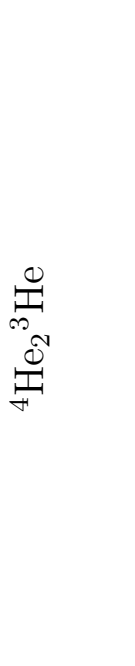 } & TTY & $-709(5)$ & $-14.23(34)$ \\
\hline & TTY+Ret & $-686(4)$ & $-13.37(15)$ \\
\hline & HFDB & $-761(6)$ & $-17.07(15)$ \\
\hline & HFDB+V3AT & $-757(4)$ & $-16.96(30)$ \\
\hline & $\mathrm{HFDB}+\mathrm{V} 3 \mathrm{BM}$ & $-756(4)$ & $-17.04(14)$ \\
\hline & HFDB+V3DDDJ & $-760(5)$ & $-16.98(11)$ \\
\hline & HFDB+Ret & $-733(4)$ & $-15.96(14)$ \\
\hline & $\mathrm{SAPTSM} \pm \varsigma$ & $-761(4)$ & $-17.16(14) \pm_{0.3}^{0.4}$ \\
\hline & SAPTSM+Ret $\pm \varsigma$ & $-736(4)$ & $-16.11(11) \pm_{0.2}^{0.1}$ \\
\hline & HFDB-2cC6 & $-432(4)$ & $-3.3(2)$ \\
\hline & HFDB-1cC6 & $-595(3)$ & $-9.3(2)$ \\
\hline & HFDB-5mC6 & $-690(9)$ & $-12.9(2)$ \\
\hline
\end{tabular}

considered models of three-body interactions proved to have a tiny effect, less than $1 \mathrm{mK}$ and $0.2 \mathrm{mK}$ for ${ }^{4} \mathrm{He}_{3}$ and ${ }^{4} \mathrm{He}_{2}{ }^{3} \mathrm{He}$, respectively. Therefore we decided not to consider them for $V_{\mathrm{BO}}$ models other than HFDB.

Many trimer properties have already been studied using different methods and interatomic potentials. Our estimates of $E_{2}, E_{3}, a_{\mathrm{s}}$ and $r_{\mathrm{e}}$ are in excellent agreement with recent results, e.g. Roudnev and Cavagnero's numerical solutions [32] of Faddeev equations for HFDB and TTY models of interactions. Bressanini [36] reported very precise DMC estimates of $E_{2}$ and $E_{3}$ obtained for the TTY model, equal to our results within error bars. Furthemore, he compared ${ }^{4} \mathrm{He}_{2}{ }^{3} \mathrm{He}$ energies computed with various methods and potentials. The results from [36] obtained using the SAPTSM and SAPTSM+Ret potentials, reported only for ${ }^{4} \mathrm{He}_{2}{ }^{3} \mathrm{He}$, are also in agreement with 
our calculation. Recently Suno, Hiyama and Kamimura 38] studied both helium trimers using the SAPTSM potential with retardation and three-body corrections. Their SAPTSM-predicted energies $E_{3}$ obtained by the adiabatic hyperspherical representation method are up to the s-errors equal to our DMC results, while their estimates using the Gaussian expansion method predict somewhat weaker binding. On the one hand, more recent results of Suno [39], obtained using the SAPTSM model in slow variable discretization approach, are within error bars equal to ours. In agreement with our results, they [38] got more significant decrease of binding when the retardation is included than with inclusion of the three-body term. The model of three-body interactions [40] which they considered was different than ours, but had similar effect on binding as our V3BM model added to the HFDB potential, i.e., a tiny reduction of binding.

Hiyama and Kamimura [41] reported the binding in the case of the currently most accurate potential PCKLJS, and obtained for ${ }^{4} \mathrm{He}_{3} E_{3}=-131.84 \mathrm{mK}$ and average potential energy $E_{p}=-1825.8 \mathrm{mK}$. In agreement with effects of SAPTSM corrections that we discussed previously, their values are between our estimates reported in table 2 for the SAPTSM and SAPTSM+Ret models.

The last three rows in table 2 report the results of the three HFDB-rC6 models, whose consideration was stimulated by the analysis of the experimental results of the trimers' structure. They reduced the energies from about 10 to almost $40 \mathrm{mK}$ in the case of ${ }^{4} \mathrm{He}_{3}$ (3 to $13 \mathrm{mK}$ in the case of ${ }^{4} \mathrm{He}_{2}{ }^{3} \mathrm{He}$ ). Similarly to the case of ${ }^{4} \mathrm{He}_{2}$, the reduction of $C_{6}$ by $2 \%$ appears unrealistic.

\subsection{Structural properties}

Challenged by the recent experimentally measured [13, 18, 19] distributions of helium trimers ${ }^{4} \mathrm{He}_{3}$ and ${ }^{4} \mathrm{He}_{2}{ }^{3} \mathrm{He}$, in addition to energy, we determined their structure using different potential models. Our goal was to evaluate which potential model gives theoretical distributions which fit the experimental data best. In order to extract exact ground state values from the DMC sampled positions we used pure estimators. Among the three-body corrections, we excluded the V3BM and V3DDDJ models because of their weaker influence on the binding energy (table 2). Results for the distribution functions are shown in figures 3, 4, 5, and 6. Functions obtained using various potential models are distinguished by different line types and widths.

In figure 3, we compare the experimental [13, 18] and our theoretically estimated ${ }^{4} \mathrm{He}-{ }^{4} \mathrm{He}$ pair distribution functions $P(r)$ of the trimer ${ }^{4} \mathrm{He}_{3}$. Results evaluated using $\mathrm{TTY}+$ Ret potential are not shown for clarity, because they are equal to results obtained using TTY and HFDB-5mC6 within the error bars. Also HFDB+Ret potential results are within error bars equal to the estimates of SAPTSM+Ret and HFDB+V3AT. Two independent experimental measurements [13, 18] and our theoretical DMC results obtained with HFDB, HFDB+V3AT, SAPTSM+Ret and TTY are thus compared in the upper panel of figure 3. Our TTY results are in agreement with TTY 


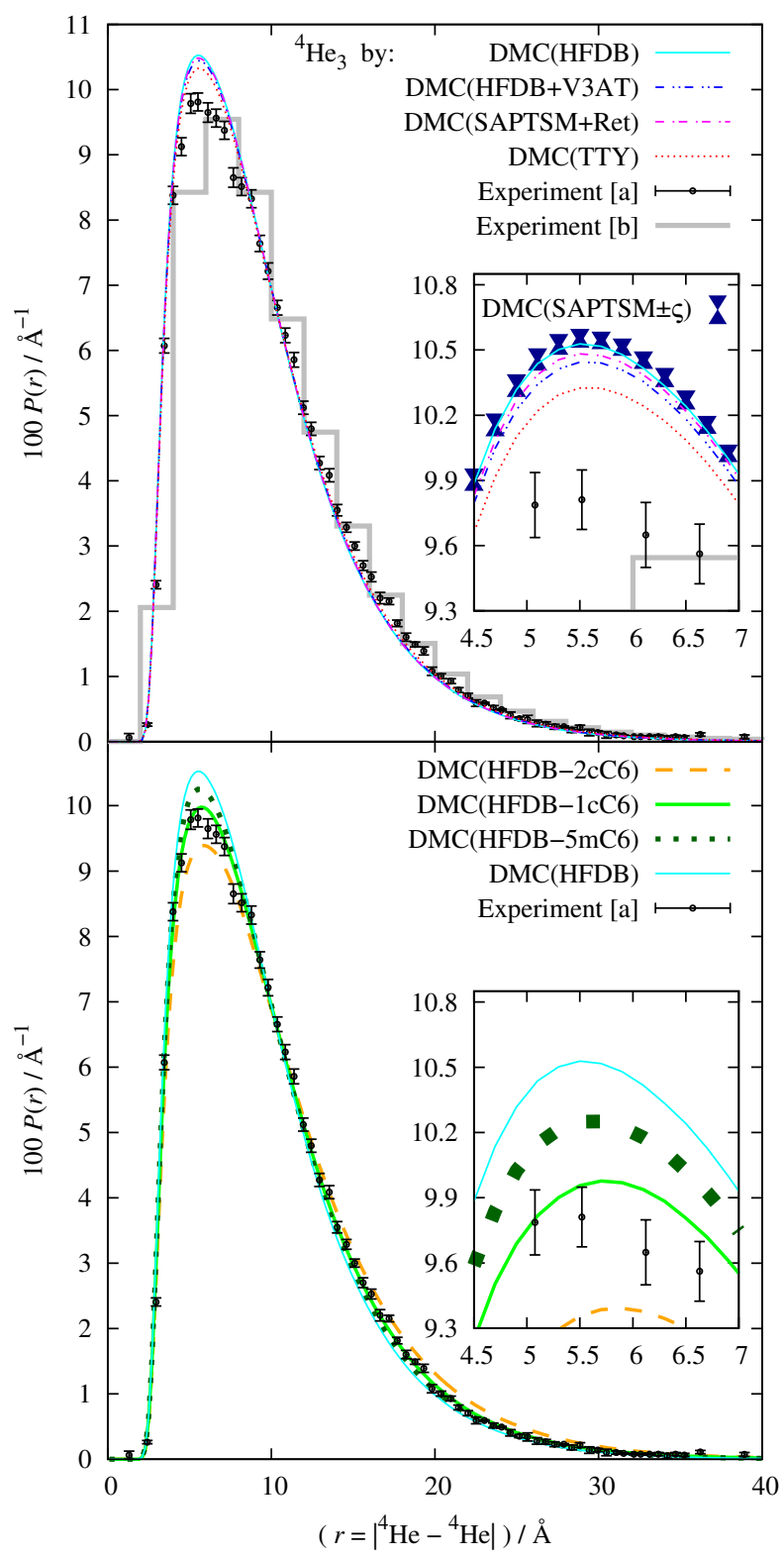

Figure 3. DMC distributions $P(r)$ of ${ }^{4} \mathrm{He}^{-}{ }^{4} \mathrm{He}$ separations $r$ in ${ }^{4} \mathrm{He}_{3}$ using different potential models (section 2.1), normalized to $\int P(r) \mathrm{d} r=1$ and compared to the measurements $[\mathrm{a}]=[13]$ and $[\mathrm{b}]=[18$. Error bars of the DMC values correspond to the triangle size in the inset.

distributions given by Voigtsberger et al [13], but given here in a smoother and more precise form. $P(r)$ was calculated also in [36] but using an approximate extrapolated estimator. Due to their accurate optimization of the trial wave function, and when this approximate estimate is properly normalized, we observed a good agreement with our TTY prediction. Furthermore very recently [39], $P(r)$ was calculated for the SAPTSM potential using the slow variable discretization method for solving the Schrödinger equation in hyperspherical coordinates. If one properly renormalizes the data from the latter work, agreement with the values reported here is observed. It is worth noting that 
in [39] comparison between ground-state theoretical and experimental data for $P(r)$ is not carried out. On the one hand, the comparison made in [13] for TTY model appeared perfect because the maxima of theoretical and experimental data were adjusted to 1 .

Theoretical models reported here predict almost the same tail, but slightly different from experimental measurements. Differences are most pronounced around the correlation peak which is zoomed in the inset. Error bars are similar for different models, thus shown only for SAPTSM: SAPTSM $+\varsigma$ and SAPTSM- $\varsigma$ plotted with upward and downward triangles, respectively. Statistical $\sigma$ error bars correspond to the size of each symbol. It is similar to the $\varsigma$ error bar of the SAPTSM results which corresponds to the distance between oppositely directed triangles. Thus SAPTSM and HFDB predict the same distribution function, even equal up to the two error bars to the distribution functions for HFDB+V3AT and SAPTSM+Ret. TTY distribution function predicts a somewhat lower peak, but not as low as experimentally measured. On the one hand, differences between the experimental data of the two reported measurements [13, 18] are not negligible. They are especially significant around the correlation peak, partly because of large differences in $r$-uncertainty resulting from the large size of the $r$-bin in one of the measurements.

A lower correlation peak and a tiny slower decay of the tail of the experimental $P(r)$ indicate a slightly weaker interaction between helium atoms. In order to investigate if agreement could be achieved by modifying $C_{6}$, we constructed the HFDB-rC6 models introduced in the previous section. The comparison of $P(r)$ obtained using these models and experimental data is presented in the bottom panel of figure 3. By weakening the dispersion coefficient $C_{6}$ in the HFDB model, the $P(r)$ correlation peak goes down and the tail raises. Reduction of $C_{6}$ by $0.5 \%$, resulted in $P(r)$ which reached experimental data up to two error bars, while reduction by $1 \%$ reproduced experimental data. Reducing $C_{6}$ even more, by $2 \%$, the resulting $P(r)$ underestimates the experimental peak and overestimates the tail.

In the top subfigure of figure 4 we compare our theoretical ${ }^{4} \mathrm{He}^{-}{ }^{4} \mathrm{He}$ pair distribution functions $P(r)$ of the trimer ${ }^{4} \mathrm{He}_{2}{ }^{3} \mathrm{He}$, while ${ }^{4} \mathrm{He}^{3} \mathrm{He}$ pair distributions are shown in the middle and the bottom subfigure. $P(r)$ are normalized to 1 in the top and the middle subfigure, while their peaks are set to 1 in the bottom subfigure in order to make a better comparison with the only available experimental data [13]. Top and middle subfigures show similar behavior of $P(r)$, as seen in figure 3 for ${ }^{4} \mathrm{He}_{3}$, but with more pronounced differences between models in the case of ${ }^{4} \mathrm{He}_{2}{ }^{3} \mathrm{He}$. The exception is the V3AT correction which has negligible effect on $P(r)$ in ${ }^{4} \mathrm{He}_{2}{ }^{3} \mathrm{He}$, and TTY distributions which separate from TTY+Ret and HFDB-5mC6. Reduction of $C_{6}$ leads to significant stretching of the cluster, so it is not shown for the HFDB-2cC6 model. Regardless of the potential model, two ${ }^{4} \mathrm{He}$ atoms are on average closer than ${ }^{4} \mathrm{He}-{ }^{3} \mathrm{He}$. Due to large experimental error bars and adjustment of the peak to 1 , in the bottom subfigure it is not clearly visible which potential is better. Only the first experimental value clearly stands out from all theoretical predictions due to a large experimental $r$-step. Opposite to the predictions for ${ }^{4} \mathrm{He}_{3}$, in ${ }^{4} \mathrm{He}_{2}{ }^{3} \mathrm{He}$ the reduction of $C_{6}$ by $0.5 \%$ fits experimental 


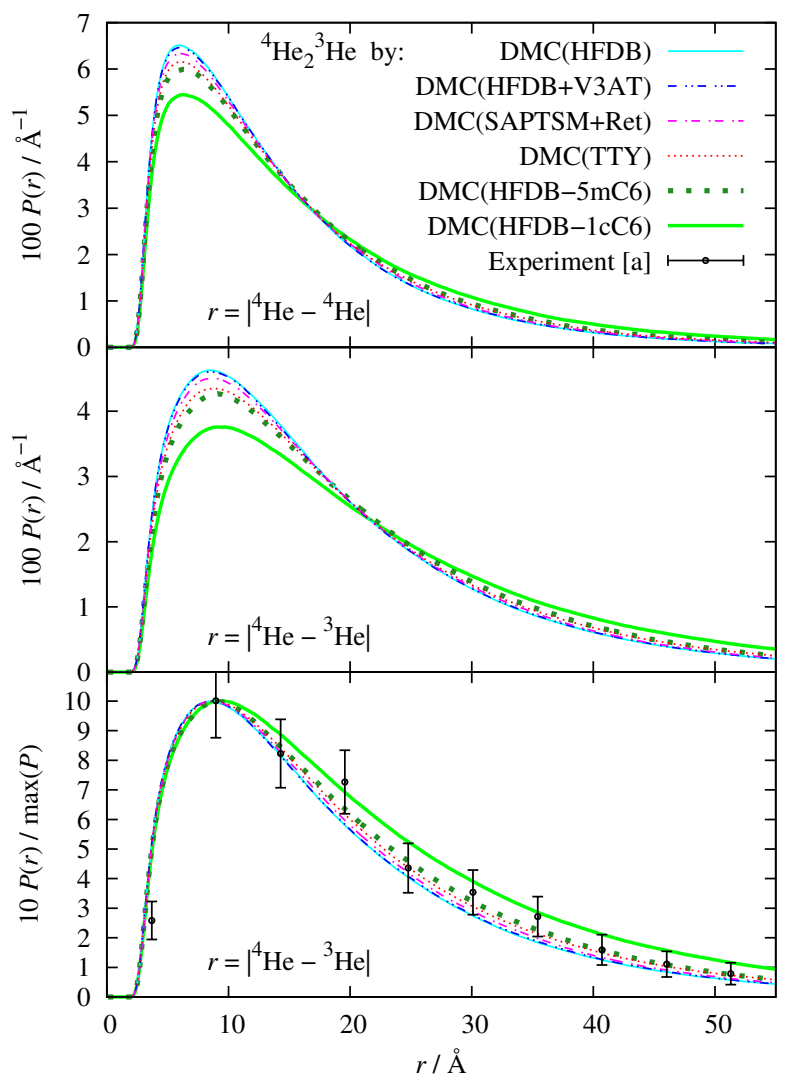

Figure 4. Distribution $P(r)$ of ${ }^{4} \mathrm{He}-{ }^{4} \mathrm{He}$ (top) and ${ }^{4} \mathrm{He}-{ }^{3} \mathrm{He}$ (middle and bottom) separations $r$ in ${ }^{4} \mathrm{He}_{2}{ }^{3} \mathrm{He}$ calculated in this work by DMC-pure estimators using different potential models (section 2.1). In the top and middle subfigures $P(r)$ are normalized to $\int P(r) \mathrm{d} r=1$, while in the bottom all correlations peak are set to 1 in order to compare calculated with measured values $[\mathrm{a}]=13$.

data better than the $1 \%$ reduction. Estimates of $P(r)$ for some models also appeared in recent literature [36, 39]. Compared to our results, properly renormalized Suno's SAPTSM results [39] slightly overestimate the ${ }^{4} \mathrm{He}-{ }^{3} \mathrm{He}$ correlation peak. On the one hand, Bressanini's TTY results [36] significantly underestimate all correlation peaks, being closest to our results obtained for HFDB-1cC6. In [36] it was argued that the high quality of wave function optimization is sufficent to ensure that, even using an extrapolated estimate, exact result for $P(r)$ can be extracted from a linear time-step DMC method. In comparison to the method used in [36], in the present work we used faster converging DMC method, i.e. second order in the time step, poorer trial wave function, but a pure algorithm which enables complete removal of the trial wave function bias from $P(r)$, which we confirmed using different trial wave functions.

In figure 5, density profiles $\rho(r)$ with $r$ the distance to the center of mass are shown for different interaction potentials. The top subfigure shows $\rho\left({ }^{4} \mathrm{He}\right)$ in ${ }^{4} \mathrm{He}_{3}$, middle $\rho\left({ }^{4} \mathrm{He}\right)$ in ${ }^{4} \mathrm{He}_{2}{ }^{3} \mathrm{He}$ and bottom $\rho\left({ }^{3} \mathrm{He}\right)$ in ${ }^{4} \mathrm{He}_{2}{ }^{3} \mathrm{He}$. One can notice similar behavior as observed in the corresponding $P(r)$. Unfortunately, there are no experimental data which could be compared. There are some theoretical estimates of $\rho(r)$ for TTY in 


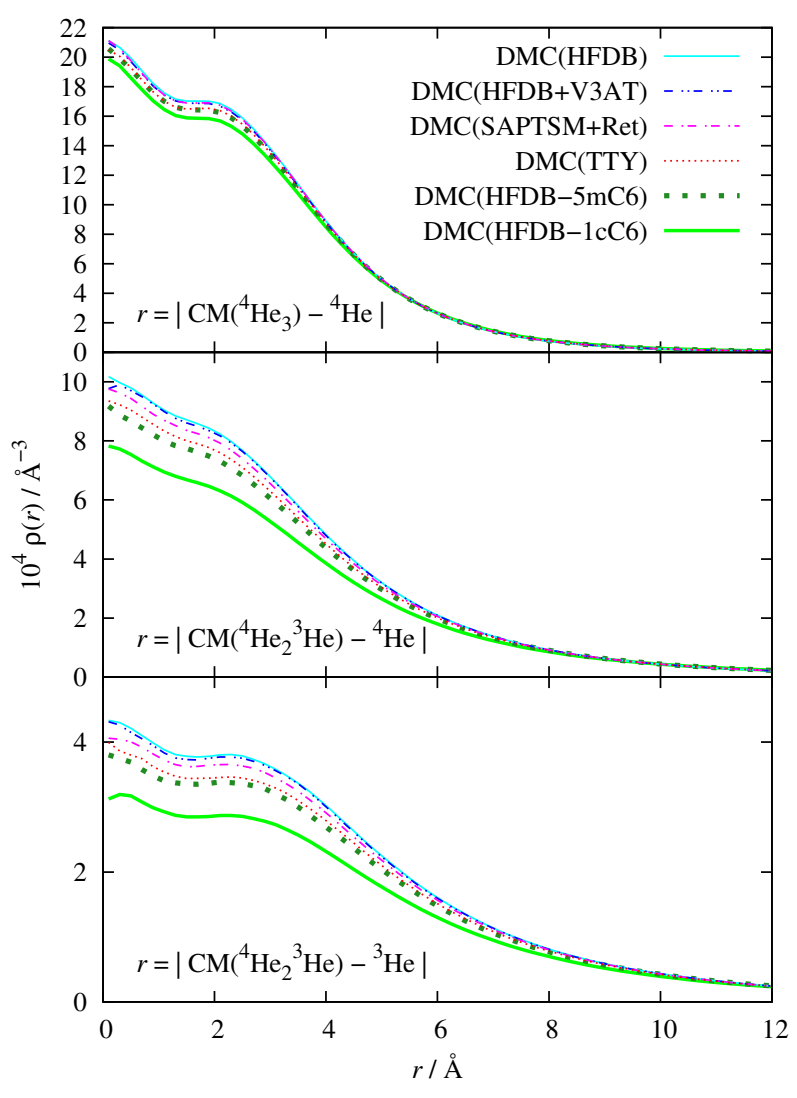

Figure 5. Density profiles $\rho(r)$ with respect to the center of mass $(\mathrm{CM})$ calculated in this work by DMC-pure estimators using different potential models (section 2.1), and normalized to $4 \pi \int \rho(r) r^{2} \mathrm{~d} r=1$. The top subfigure stands for ${ }^{4} \mathrm{He}$ in ${ }^{4} \mathrm{He}_{3}$, while middle and bottom ones for ${ }^{4} \mathrm{He}$ and ${ }^{3} \mathrm{He}$ in ${ }^{4} \mathrm{He}_{2}{ }^{3} \mathrm{He}$, respectively.

the literature [36, 42], but a precise comparison is not possible in the cases where tails are missing due to inconsistent information regarding normalization. When $\rho\left({ }^{4} \mathrm{He}\right)$ in ${ }^{4} \mathrm{He}_{3}$ from [36] is properly renormalized, agreement with present results is obtained. Furthermore, $\rho\left({ }^{4} \mathrm{He}\right)$ in ${ }^{4} \mathrm{He}_{2}{ }^{3} \mathrm{He}$ given in [36, 42] are even visually extremely different. Estimate from [42] is similar to the ours, while estimate from [36] differs a lot, e.g. predicts that ${ }^{4} \mathrm{He}$ can not be close to the center of mass while there is no physical reason for such a behavior.

Angular distribution functions are presented for different potentials in figure 6. The top subfigure shows the distribution $P(\vartheta)$ of corner angle $\vartheta=\measuredangle\left({ }^{4} \mathrm{He}-{ }^{4} \mathrm{He}-{ }^{4} \mathrm{He}\right)$, middle of $\vartheta=\measuredangle\left({ }^{4} \mathrm{He}-{ }^{4} \mathrm{He}-{ }^{3} \mathrm{He}\right)$ and bottom of $\vartheta=\measuredangle\left({ }^{4} \mathrm{He}-{ }^{3} \mathrm{He}-{ }^{4} \mathrm{He}\right)$. Experimental data are taken from [13], but here normalized to 1, and shown with error bars. Error bars of the theoretical data (this work) are of the same order of magnitude as the line width. In the top subfigure all theoretical estimates are almost the same. The most significant difference with experiment is the sharp peak of experimental data that neither theoretical model predicts. In the middle subfigure, experimental data are scattered around theoretical estimates. Even the reduction of $C_{6}$ by $2 \%$ makes no significant difference in the predictions of $P(\vartheta)$, while differences are 


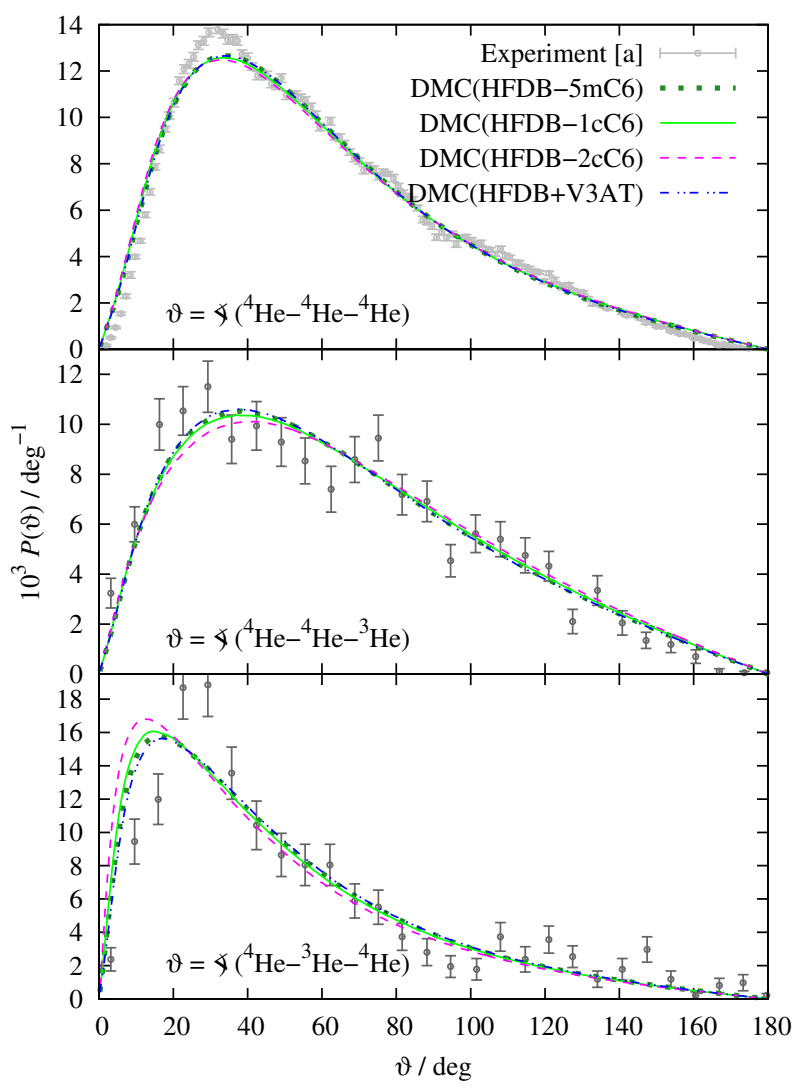

Figure 6. Distribution $P(\vartheta)$ in trimers ${ }^{4} \mathrm{He}_{3}$ and ${ }^{4} \mathrm{He}_{2}{ }^{3} \mathrm{He}$ of corner angle $\vartheta$, in top subfigure $\measuredangle\left({ }^{4} \mathrm{He}-{ }^{4} \mathrm{He}-{ }^{4} \mathrm{He}\right)$, in middle $\measuredangle\left({ }^{4} \mathrm{He}-{ }^{4} \mathrm{He}-{ }^{3} \mathrm{He}\right)$, and in bottom $\measuredangle\left({ }^{4} \mathrm{He}-{ }^{3} \mathrm{He}-{ }^{4} \mathrm{He}\right)$ using different potential models (section 2.11) and compared with renormalized measured values $[\mathrm{a}]=[13]$. All distributions are normalized to $\int P(\vartheta) \mathrm{d} \vartheta=1$.

clearly pronounced in the case of $P(r)$ (see figure 3). From visual inspection it is not possible to conclude which potential model leads to the distribution that fits better the experimental data. In the bottom subfigure, similar behavior is noticeable, but with more scattered experimental data. Again neither theoretical model predicts the peak to be as sharp as extracted [13] from experimentally measured data. Recently, theoretical estimates of $P(\vartheta)$ also for the models TTY [36] and SAPTSM [39] have been published. When comparing, one needs to be careful due to wrong normalizations. When properly renormalized, agreement with present results is obtained.

In order to numerically evaluate which potential model makes better predictions we chose $P_{i}=P\left(r_{i}\right)$ in ${ }^{4} \mathrm{He}_{3}$, because only these experimental data $P^{\exp }$ are given [13] with known norm and the smallest error bars $\sigma\left(P_{i}^{\text {exp }}\right)$ relative to the differences between our theoretical model predictions $P^{\mathrm{dmc}}(r)$. We made numerical estimates of differences $\Delta P$ between experimental and theoretical predictions. Different definitions of differences were used

$$
\langle\Delta P\rangle=\frac{1}{n} \sum_{i}^{n}\left(P_{i}^{\mathrm{dmc}}-P_{i}^{\exp }\right)^{2}
$$


Table 3. Differences $\Delta P$, between $\operatorname{DMC}(V)$ pure estimates and experimental data digitalized from figure 1c in [13, calculated using (6), (7) and (8).

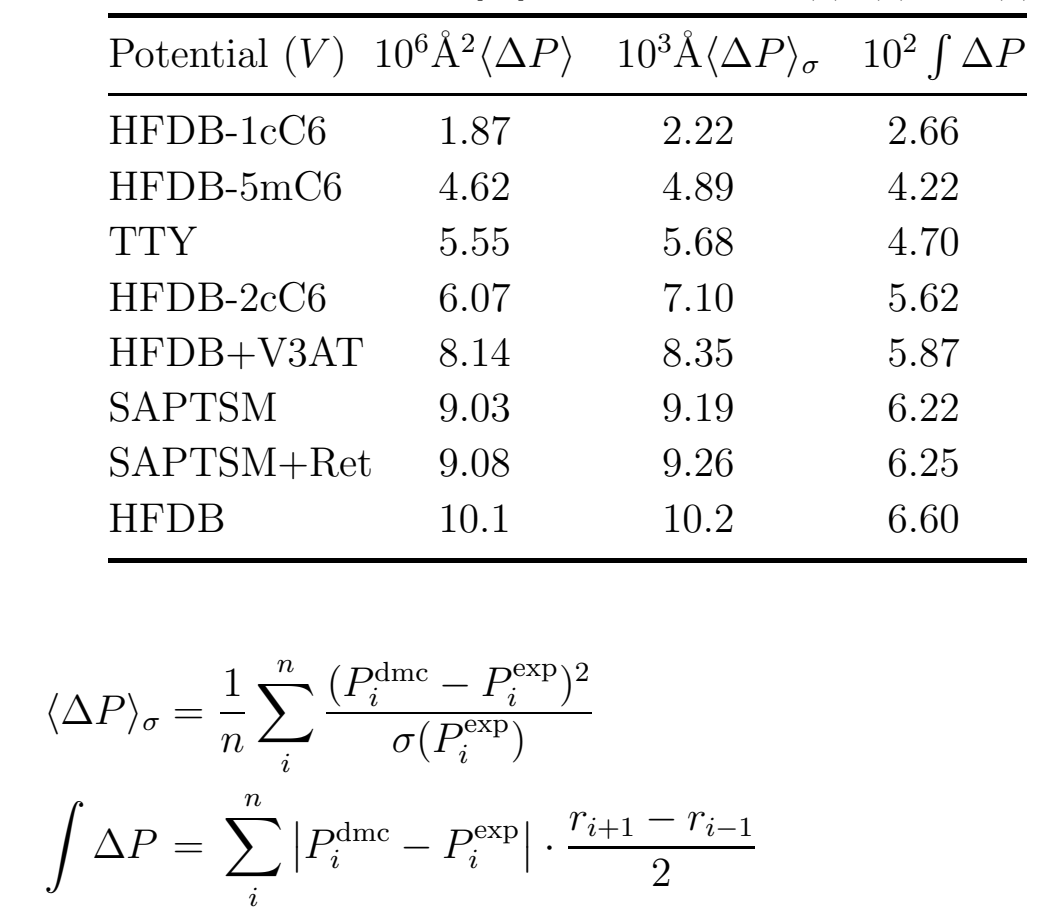

where a linear interpolation was used to calculate $P_{i}^{\mathrm{dmc}}$ in each experimental point $r_{i}$. Digitalized experimental data are not good enough in areas where symbols, errobars and axes cannot be clearly distinguished, so we approximated $P^{\mathrm{dmc}} \approx P^{\exp }$ for $r>30 \AA$. Definitions (6), (7) and (8) have returned the same ascending sorted list of potential models (from the best to the worst): HFDB-1cC6, HFDB-5mC6, TTY, HFDB-2cC6, HFDB+V3AT, SAPTSM, SAPTSM+Ret, HFDB. Calculated values are given in table 3 . A similar list could be obtained just by visual comparison of different results in figure 3 .

\subsection{Universal scaling}

In a previous work, we established [16] both the more convenient energy-size scaling and the universal lines which trimer halo states do follow. The size of a system was measured [16] by the root-mean-square hyperradius $\rho$,

$$
m \rho^{2}=\frac{1}{M} \sum_{i<k} m_{i} m_{k}\left\langle r_{i k}^{2}\right\rangle,
$$

where $m$ is an arbitrary mass unit, $m_{i}$ the particle mass of species $i, M$ the total mass of the system, and $\left\langle r_{i k}^{2}\right\rangle$ the mean square distance between particles $i$ and $k$. Values of $\left\langle r_{i k}^{2}\right\rangle$ extracted by the pure estimators from the DMC-sampled positions are given in table 4. The mean square distance between ${ }^{4} \mathrm{He}$ atoms in ${ }^{4} \mathrm{He}_{3}$, obtained by the most recent $V_{\mathrm{BO}}$ potential SAPTSM is in agreement with the value given in [41], estimated using the most detailed post $V_{\mathrm{BO}}$ potential, PCKLJS. Universal lines from [16] are shown in figure 7. All-bound trimer type is presented by a dotted line which, when binding is 
Table 4. Mean square distances $\left\langle r_{44}^{2}\right\rangle$ between ${ }^{4} \mathrm{He}-{ }^{4} \mathrm{He}$ and $\left\langle r_{43}^{2}\right\rangle$ between ${ }^{4} \mathrm{He}-{ }^{3} \mathrm{He}$ in clusters ${ }^{4} \mathrm{He}_{3}$ and ${ }^{4} \mathrm{He}_{2}{ }^{3} \mathrm{He}$. Standard deviations are $1-3 \%$ of the corresponding quantity.

\begin{tabular}{lcccc}
\hline \multirow{2}{*}{ Potential } & ${ }^{4} \mathrm{He}_{3}$ & & \multicolumn{2}{c}{${ }^{4} \mathrm{He}_{2}{ }^{3} \mathrm{He}$} \\
\cline { 2 - 3 } \cline { 5 - 5 } & $\left\langle r_{44}^{2}\right\rangle / \AA^{2}$ & $\left\langle r_{44}^{2}\right\rangle / \AA^{2}$ & $\left\langle r_{43}^{2}\right\rangle / \AA^{2}$ \\
\hline SAPTSM & 116 & & 330 & 540 \\
HFDB & 117 & & 326 & 560 \\
HFDB+V3AT & 117 & & 336 & 550 \\
SAPTSM+Ret & 117 & & 356 & 590 \\
HFDB+Ret & 118 & & 360 & 608 \\
TTY & 120 & & 368 & 635 \\
TTY+Ret & 121 & & 384 & 663 \\
HFDB-5mC6 & 123 & & 404 & 680 \\
HFDB-1cC6 & 128 & & 482 & 884 \\
\hline
\end{tabular}

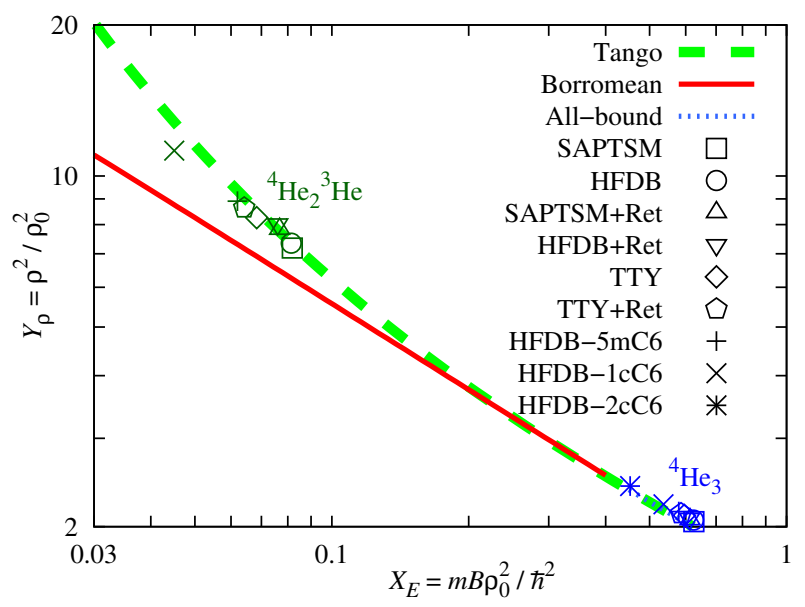

Figure 7. Absolute binding energy $B$ and size of trimers ${ }^{4} \mathrm{He}_{3}$ and ${ }^{4} \mathrm{He}_{2}{ }^{3} \mathrm{He}$ are calculated by DMC-pure estimators using different potential models (section 2.1) and scaled using definitions $X_{E}$ and $Y_{\rho}$ given in [16]. Different symbols are used to distinguish potential models. Universal lines for Borromean, tango and all-bound trimer type are fitted through data given in [16. Halo states are defined by the condition [14] $Y_{\rho} \gtrsim 2$.

decreased, passes into the Borromean type presented by solid line. Dashed line shows a departure of the tango trimer type from the joint universal line. Symbols representing scaled energy $X_{E}$ and size $Y_{\rho}$ for both helium trimers, obtained using different potential models fall on the universal lines plotted in figure 7 . Results of all presented models predict both helium trimers ${ }^{4} \mathrm{He}_{3}$ and ${ }^{4} \mathrm{He}_{2}{ }^{3} \mathrm{He}$ to be in a halo state and to follow the universal line, being spatially wider and more weakly bound the less attractive the potential is. 


\section{Summary and conclusions}

Many semi-empirical and ab initio potential models have been proposed for the interaction of helium atoms. Considering their diversity, we made a rather complete set of tests on how the interaction potentials, and their corrections, influence the ground state binding energy and structural properties of small helium clusters. The clusters most sensitive to the changes in the interaction potential were considered, dimers and trimers.

The DMC method, which was used to calculate the trimer properties, gave exact values of their studied properties within statistical error bars. The achieved binding energy statistical errors $\sigma$ were few times smaller than errors caused by the SAPTSMuncertainty $\varsigma$. Our predictions are in excellent agreement with the most recent estimates, obtained with various methods, confirming their accuracy. Structural properties were determined with $\sigma$ approximately equal to $\varsigma$.

For some models, like HFDB+V3AT, HFDB+V3BM, HFDB+V3DDDJ, TTY+Ret, HFDB+Ret, HFDB-2cC6, HFDB-1cC6, and HFDB-5mC6, we made estimates of the trimer properties for the first time. In particular, the influence of the error bar $\varsigma$ of the newest and most sophisticated He-He $V_{\mathrm{BO}}$ estimate SAPTSM and its most significant correction Ret on helium trimer structural properties, was analyzed for the first time. Influence of other post- $V_{\mathrm{BO}}$ SAPTSM-corrections ARQ embedded in the PCKLJS model were not considered because they are smaller than Ret, and therefore give trimer properties close to estimates obtained with SAPTSM and SAPTSM+Ret, which already are not so different. Furthermore, we estimated the influence of attenuated HFDB potential by reducing dispersion coefficient $C_{6}$ for $2 \%(-2 \mathrm{cC} 6), 1 \%(-1 \mathrm{cC} 6)$ and $0.5 \%(-5 \mathrm{mC} 6)$.

Among structural properties calculated in this work, angular distributions $P(\vartheta)$ are the least affected by the potential model; differences are barely visible. On the one hand, measured $P(\vartheta)$ are the most cascade-like among experimental data given in [13]. Therefore measured values cannot be used to evaluate potential models. However, even if we had very precise measurements of angular distributions, we could not use them to rate potential corrections, because many models give similar theoretical predictions.

According to theoretical estimates, some potential models, attenuations of $C_{6}$ and correction Ret could be distinguished from the density profiles $\rho(r)$ with respect to the center of mass and from the distributions of interparticle separations $P(r)$. Unfortunately, there are no measured values of $\rho(r)$, but there are some of $P(r)$. From visual comparison, specifying indistinguishable models as one set, we can sort potential models from the lowest to the highest correlation peak of $P(r)$ in ${ }^{4} \mathrm{He}_{3}$ : \{HFDB-2cC6\}, \{Experiment [18]\}, \{Experiment [13], HFDB-1cC6\}, \{HFDB$5 \mathrm{mC6}, \mathrm{TTY}+$ Ret $\},\{\mathrm{TTY}\},\{\mathrm{HFDB}+$ Ret, SAPTSM+Ret, HFDB+V3AT $\},\{\mathrm{HFDB}$, SAPTSM $\}$. Differences are more clear when $P(r)$ are compared in ${ }^{4} \mathrm{He}_{2}{ }^{3} \mathrm{He}$; only the effect of the three-body correction V3AT becomes invisible. Unfortunately there are no normalized experimental distributions in ${ }^{4} \mathrm{He}_{2}{ }^{3} \mathrm{He}$, so comparison is made setting 
all peaks to 1 . In that case HFDB-5mC6 fits the experimental data best. But this adjustment is somehow unnatural because distribution differences between models become significant in some areas where they are equal when normalized to 1 . As expected, similar comparison between theoretical models follows from the mean square interparticle distances.

In order to go beyond a simple visual comparison, we evaluated measured-calculated differences $\Delta P$ of $P(r)$ in ${ }^{4} \mathrm{He}_{3}$. In this way we got a sorted list of potential models (from the best to the worst predictor according to table 4): HFDB-1cC6, HFDB-5mC6, TTY, HFDB-2cC6, HFDB+V3AT, SAPTSM, SAPTSM+Ret, HFDB. However, the first model HFDB-1cC6 significantly underestimates dimer binding energies $E_{2}^{\prime}$ and $E_{2}^{\prime \prime}$ which follow from the two analysis [11, 28] (11a) and (5a) of the experimental data [11]. The second best HFDB-5mC6 predicts $P(r)$ in ${ }^{4} \mathrm{He}_{3}$ up to the error bar equal to the TTY+Ret results. Although HFDB-5mC6 and TTY+Ret predict distinguishable dimer binding energies, due to the large error bars in values (1a) and (5a), it is not possible to state which is better.

All our theoretical estimates predicted both helium trimers, all-bound type ${ }^{4} \mathrm{He}_{3}$ and tango type ${ }^{4} \mathrm{He}_{2}{ }^{3} \mathrm{He}$, to be in a ground halo state, although recent articles [13, 19] mention ${ }^{4} \mathrm{He}_{2}{ }^{3} \mathrm{He}$ and only excited state of ${ }^{4} \mathrm{He}_{3}$ as a halo. Both are structureless clouds. However, the less bound ${ }^{4} \mathrm{He}_{2}{ }^{3} \mathrm{He}$ is wider and more spread among different shapes (linear, isoceles, scaline, equilateral).

With development of methods and increase of computer power, theoretical estimates of helium cluster properties have become very accurate and efficient. Theoretical uncertainties are more than an order of magnitude smaller than experimental ones. Furthermore, the discrepancies between computed and measured values are a few times larger than the theoretical uncertainties. Therefore, a higher precision of experimental measurements would be welcomed to derive a more accurate rating of theoretical models. The temperature could affect the measured values and these effects are not taken into account in our theoretical estimates. Additionaly, the ground state of ${ }^{4} \mathrm{He}_{3}$ could be contaminated by a fraction of the excited state, which could explain differences between two [13, 18] experimental measurements.

In conclusion, the whole set of available measured and deduced values, from experimental helium dimer and trimers data, is in the best agreement with the theoretical predictions obtained using the potential models TTY+Ret and HFDB-5mC6, which are up to the error bars equal.

\section{Acknowledgments}

This work has been supported in part by the Croatian Science Foundation under the project number IP-2014-09-2452. J. B. acknowledge additional support by the MICINNSpain, Grant No. FIS2014-56257-C2-1-P. The computational resources of the Isabella cluster at Zagreb University Computing Center (Srce), the HYBRID cluster at the University of Split, Faculty of Science and Croatian National Grid Infrastructure (CRO NGI) were used. 


\section{References}

[1] Toennies J P, Vilesov A F and Whaley K B 2001 Physics Today 5431

[2] Toennies J P 2013 Mol Phys 1111879

[3] Whaley K B 1994 Int Rev Phys Chem 1341

[4] Sola E, Casulleras J and Boronat J 2006 Phys. Rev. B 73092515

[5] Guardiola R and Navarro J 2003 Phys. Rev. A 68055201

[6] Bressanini D and Morosi G 2004 Few-Body Systems 34131

[7] Luo F, McBane G, Kim G and Giese C F 1993 J. Chem. Phys. 983564

[8] Luo F, Giese C F and Gentry W R 1996 J. Chem. Phys. 1041151

[9] Schllkopf W and Toennies J P 1994 Science 2661345

[10] Schllkopf W and Toennies J P 1996 J. Chem. Phys. 1041155

[11] Grisenti R E, Schllkopf W, Toennies J P, Hegerfeldt G C, Khler T and Stoll M 2000 Phys. Rev. Lett. 852284

[12] Kalinin A, Kornilov O, Schllkopf W and Toennies J P 2005 Phys. Rev. Lett. 95113402

[13] Voigtsberger J et al 2014 Nature Communications 55765

[14] Jensen A S, Riisager K, Fedorov D V and Garrido E 2004 Rev. Mod. Phys. 76215

[15] Riisager K 2013 Phys. Scr. 2013 T152 014001

[16] Stipanović P, Vranješ Markić L, Bešlić I and Boronat J 2014 Phys. Rev. Lett. 113253401

[17] Efimov V 1970 Phys. Lett. 33B 563

[18] Kunitski M et al 2015 Science $\mathbf{3 4 8} 551$

[19] Kornilov O 2015 Science 348498

[20] Axilrod B M and Teller E 1943 J. Chem. Phys. 11299

[21] Casimir H B G and Polder D 1948 Phys. Rev. 73360

[22] Bruch L W and McGee I J 1973 J. Chem. Phys. 59409

[23] Aziz R A, McCourt F R W and Wong C C K 1987 Mol Phys 611487

[24] Tang K T, Toennies J P and Yiu C L 1995 Phys. Rev. Lett. 741546

[25] Ujevic S and Vitiello S A 2006 Phys. Rev. B 73 012511; Cohen M J and Murrell J N 1996 Chem Phys Lett $\mathbf{2 6 0} 371$

[26] Jeziorska M, Cencek W, Patkowski B, Jeziorski B and Szalewicz K 2007 J. Chem. Phys. 127 124303

[27] Przybytek M, Cencek W, Komasa J, ach G, Jeziorski B and Szalewicz K 2010 Phys. Rev. Lett. $\mathbf{1 0 4}$ 183003; See also supplementary material at http://linkapsorg/supplemental/101103/PhysRevLett104183003 and the errata 2012 Phys. Rev. Lett. 108129902

[28] Cencek W, Przybytek M, Komasa J, Mehl J B, Jeziorski B and Szalewicz K 2012 J. Chem. Phys. 136224303

[29] Stipanović P, Vranješ Markić L, Boronat J and Kežić B 2011 J. Chem. Phys. 134054509

[30] Boronat J and Casulleras J 1994 Phys. Rev. B 498920

[31] Casulleras J and Boronat J 1995 Phys. Rev. B 523654

[32] Roudnev V and Cavagnero M 2012 J. Phys. B: At. Mol. Opt. Phys. 45025101

[33] Jensen A S, Riisager K, Fedorov D and Garrido E 2004 Rev. Mod. Phys. 76215

[34] Robicheaux F 1999 Phys. Rev. A 601706

[35] Yamashita M T, Tomio L and Frederico T 2004 Nuclear Phys A 73540

[36] Bressanini D 2014 J. Phys. Chem. A 1186521

[37] Braaten E and Hammer H-W 2003 Phys. Rev. A 67042706

[38] Suno H, Hiyama E and Kamimura M 2013 Few-Body Systems 541557

[39] Suno H 2016 J. Phys. B: At. Mol. Opt. Phys. 49014003

[40] Cencek W, Jeziorska M, Akin-Ojo O and Szalewicz K 2007 J. Phys. Chem. A 11111311

[41] Hiyama E and Kamimura M 2012 Phys. Rev. A 85062505

[42] Bressanini D, Zavaglia M, Mella M and Morosi G 2000 J. Chem. Phys. 112717 